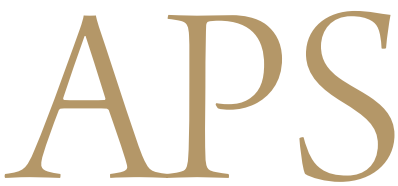

Archives of Plastic Surgery

\title{
Deep-Plane Lipoabdominoplasty in East Asians
}

\author{
June-Kyu Kim ${ }^{1}$, Jun-Young Jang ${ }^{1}$, Yoon Gi Hong ${ }^{2}$, Hyung Bo Sim³ ${ }^{3}$, Sang Hoon Sun ${ }^{3}$ \\ ${ }^{1}$ Department of Plastic and Reconstructive Surgery, Kangbuk Samsung Medical Center, Sungkyunkwan University School of Medicine, Seoul, \\ Korea; ${ }^{2}$ YeStar Plastic Surgery, Shanghai, China; ${ }^{3}$ BR Plastic Surgery, Seoul, Korea
}

Background The objective of this study was to develop a new surgical technique by combining traditional abdominoplasty with liposuction. This combination of operations permits simpler and more accurate management of various abdominal deformities. In lipoabdominoplasty, the combination of techniques is of paramount concern. Herein, we introduce a new combination of liposuction and abdominoplasty using deep-plane flap sliding to maximize the benefits of both techniques.

Methods Deep-plane lipoabdominoplasty was performed in 143 patients between January 2007 and May 2014. We applied extensive liposuction on the entire abdomen followed by a sliding flap through the deep plane after repairing the diastasis recti. The abdominal wound closure was completed with repair of Scarpa's fascia.

Results The average amount of liposuction aspirate was 1,400 $\mathrm{mL}(700-3,100 \mathrm{~mL})$, and the size of the average excised skin ellipse was $21.78 \times 12.81 \mathrm{~cm}$ (from $15 \times 10$ to $25 \times 15 \mathrm{~cm}$ ). There were no major complications such as deep-vein thrombosis or pulmonary embolism. We encountered 22 cases of minor complications: one wound infection, one case of skin necrosis, two cases of undercorrection, nine hypertrophic scars, and nine seromas. These complications were solved by conservative management or simple revision.

Conclusions The use of deep-plane lipoabdominoplasty can correct abdominal deformities more effectively and with fewer complications than traditional abdominoplasty.

\section{Keywords Lipectomy / Abdominoplasty / Abdominal wound closure techniques}

Correspondence: June-Kyu Kim Department of Plastic and Reconstructive Surgery, Kangbuk Samsung Medical Center, Sungkyunkwan University School of Medicine, 29 Saemunan-ro, Jongnogu, Seoul 03181, Korea Tel: +82-2-2001-2178 Fax: +82-2-2001-2177 E-mail: kokoro72@naver.com

Received: 31 Dec 2015 • Revised: 4 Apr 2016 - Accepted: 12 Apr 2016

pISSN: 2234-6163 • elSSN: 2234-6171 • http://dx.doi.org/10.5999/aps.2016.43.4.352 • Arch Plast Surg 2016;43:352-359

No potential conflict of interest relevant to this article was reported.

\section{INTRODUCTION}

There has been a recent increase in the number of patients interested in correcting body contour problems. Many conditions including obesity, severe weight loss, and pregnancy cause changes in the body contour, including that of the abdominal region. The increased need for abdominal plastic surgery associated with bariatric surgery has led to the intensive development of a range of surgical techniques [1].

Liposuction and abdominoplasty have each developed their own distinct surgical concepts, techniques, and equipment. The effective combination of these two different techniques is of paramount concern in the correction of abdominal deformities, including excessive skin, excessive fat, diastasis recti, and loss of the waist line. Wide abdominal flap undermining is directly associated with flap morbidities such as necrosis, seroma, and hematoma. Other complications, such as scar hypertrophy and unexpected scar position, require special attention and surgical skill [2].

We combined liposuction of the superficial and deep fat layers of the entire abdomen, full-thickness resection of the infraumbilical ellipse, mobilization of the abdominal flap through the 
deep plane, and fascial repair. We named this technique deepplane lipoabdominoplasty (DPLA).

\section{METHODS}

From January 2007 to May 2014, we performed DPLA in 143 patients to correct their abdominal contour. Four of the patients were men and 139 were women.

Ninety-seven patients suffered from abdominal changes after pregnancy, and abdominal bulging with striae gravidarum was the major postpartum problem. Twenty-four of those 97 patients had an abdominal vertical scar, 11 had a surface irregularity after liposuction surgery from other clinics, and 5 seriously obese patients (with a body mass index over $30 \mathrm{~kg} / \mathrm{m}^{2}$ ) underwent surgery to treat abdominal pendulum.

\section{Operating techniques}

\section{Design}

A fusiform design was marked on the abdomen for skin excision. There are no standard practices for designing the ellipse, and the lateral point was chosen based on each patient's needs. The proposed lower abdominal incision was marked under the patient's underwear, and utilizing the pinch technique, the superior extent of the incision was approximated with minimal to no tension. It is beneficial to mark the area beneath the patient's clothing. If the patient prefers to wear low-cut jeans, it is important to hide the scar under the clothing.

\section{Infiltration}

The abdomen was infiltrated with tumescent fluid using the su-

\section{Fig. 1. External laser irradiation}

External laser irradiation was performed on the abdominal surface. The immediate effect of the laser is to produce temporary pores in the cell membranes of adipocytes, allowing fat to migrate into the extracellular space. This technique speeds up fat removal.

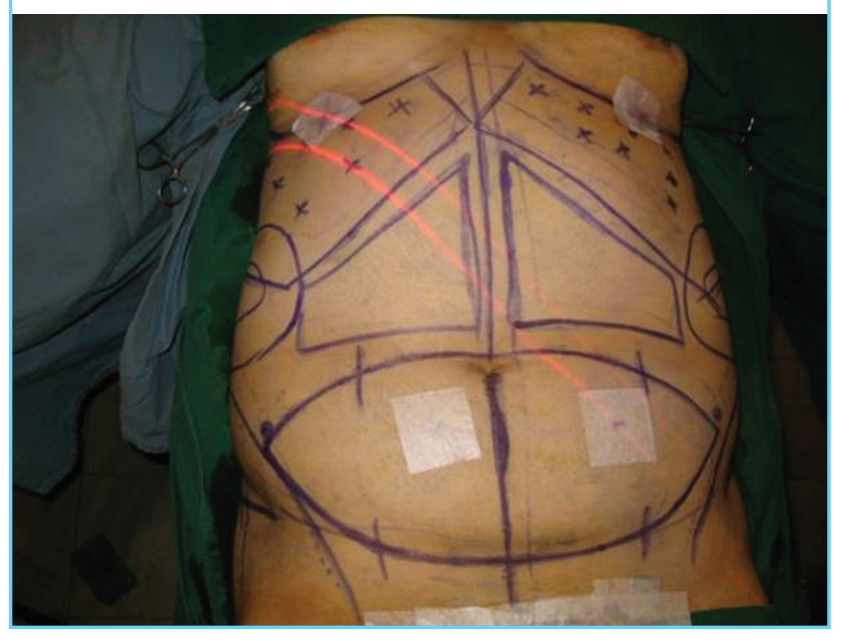

perwet technique. Each liter of tumescent solution consisted of 1 liter of lactated Ringer's solution, $10 \mathrm{~mL}$ of $1 \%$ lidocaine, and 1 $\mathrm{mL}$ of 1:1,000 epinephrine. The total lidocaine dose was limited to a maximum of $35 \mathrm{mg} / \mathrm{mL}$.

\section{External laser}

After fluid infiltration, liposuction efficiency was enhanced by irradiating the abdominal surface with an EML (Erchonia Medical Co., McKinney, TX, USA) or Laserslim (Nanum Medical Inc., Seoul, Korea), for approximately 15 minutes (Fig. 1).

\section{Liposuction}

With the patient in the supine position, liposuction was performed over the subcostal area beneath the breast and on the infraumbilical region. A 3-4 mm cannula was used to remove the fat from the superficial and deep layers of the flank and lower abdomen (Fig. 2).

\section{Skin excision and umbilical isolation}

After completing the liposuction procedure, tension on the abdominal skin was relieved enough to be easily pulled down by pinching. A full-thickness resection of the infraumbilical abdominal ellipse, including Scarpa's fascia and the deep fat layer, was performed after umbilical isolation, which is consistent with traditional abdominoplasty. A small amount of deep fat was preserved over the deep fascia, especially in the bilateral inguinal regions (Fig. 3).

\section{Rectus plication}

Median abdominal undermining was performed between the medial borders of the rectus abdominis muscle. Special care was taken not to pass beyond the borders. This strategy is similar to

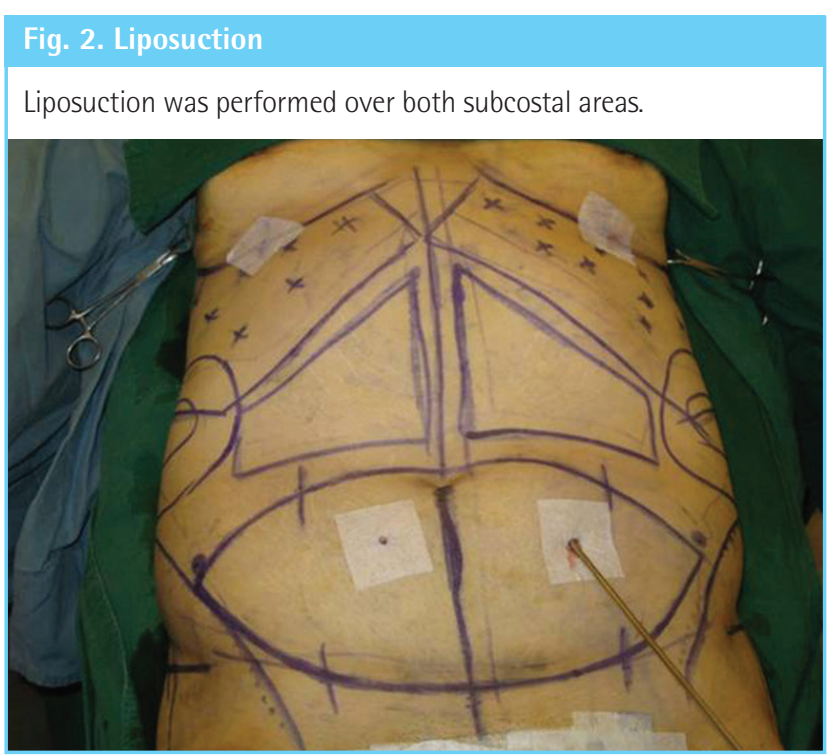


the selective undermining of Saldanha's technique [3]. If the infraumbilical diastasis is repaired first, the width of the supraumbilical diastasis may be narrowed, making the medial border of the rectus more obvious in the upper abdomen, and thereby securing a clear visual field in the narrow tunnel (Fig. 4).

Flap sliding

If liposuction was sufficient and even, sliding of the abdominal flap was performed without difficulty. The sliding plane was below Scarpa's fascia (Fig. 5).

\section{Wound closure}

In contrast to the traditional approach, surgical closure must be-

\section{Fig. 3. Abdominal tissue removal}

The abdominal tissue was removed, including Scarpa's fascia and the deep fat, except over the bilateral inguinal region. Umbilical isolation was also performed.

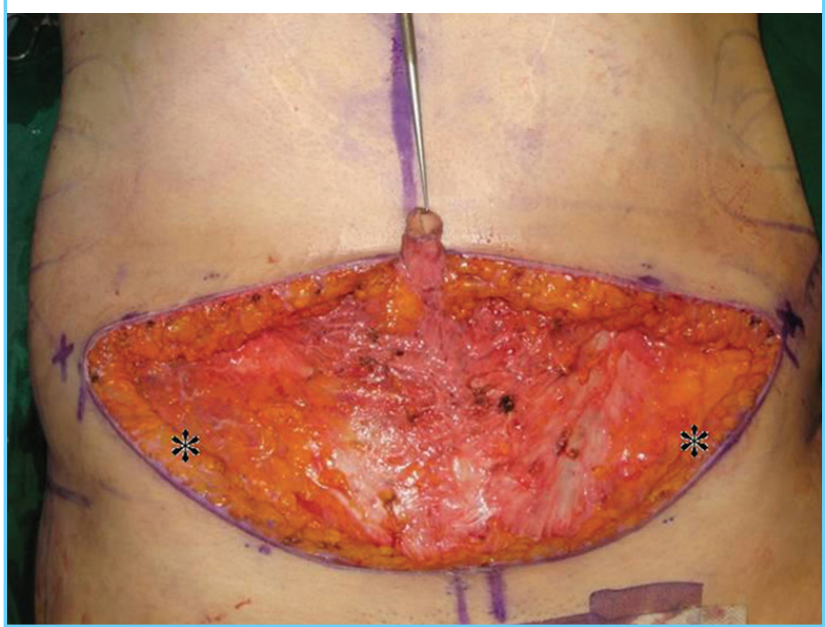

gin from the lateral region and move medially to ensure symmetry without creating a dog-ear deformity. The Scarpa's fascia was repaired first, and then the subcutaneous tissue and dermis were closed securely. This approach was used to reduce the tension on the midline skin closure by placing more tension laterally (Fig. 6).

\section{Umbilical transposition}

A 1.0-2.0 cm vertical elliptical excision for the abdominal flap was made in the midline overlying the buried umbilicus. A path was then created by blunt dissection for the stalk. The point of the new umbilicus was above the level of the anterior superior iliac spine or $10-15 \mathrm{~cm}$ over the superior border of the pubic tubercle. Three point fixation sutures at 3 o'clock, 6 o'clock, and 9 o'clock were placed on the subcutaneous layer of the abdominal flap and the umbilicus through the fascia of the surrounding abdominal wall.

\section{Drain insertion}

We used a continuous Jackson-Pratt drain for drainage over the course of three or seven days.

\section{Skin closure}

After securing the fascia and dermal suture, we applied skin bond (Histoacryl, B. Braun, Aesculap, Germany) along the incisional wounds (Fig. 7).

\section{RESULTS}

We corrected abdominal deformities without life-threatening

\section{Fig. 4. Infraumbilical diastasis repair}

(A) Infraumbilical diastasis was repaired first, providing a clear visual field in the upper abdomen and making the rectus muscle border more clearly visible. (B) Schematic illustration of this procedure.
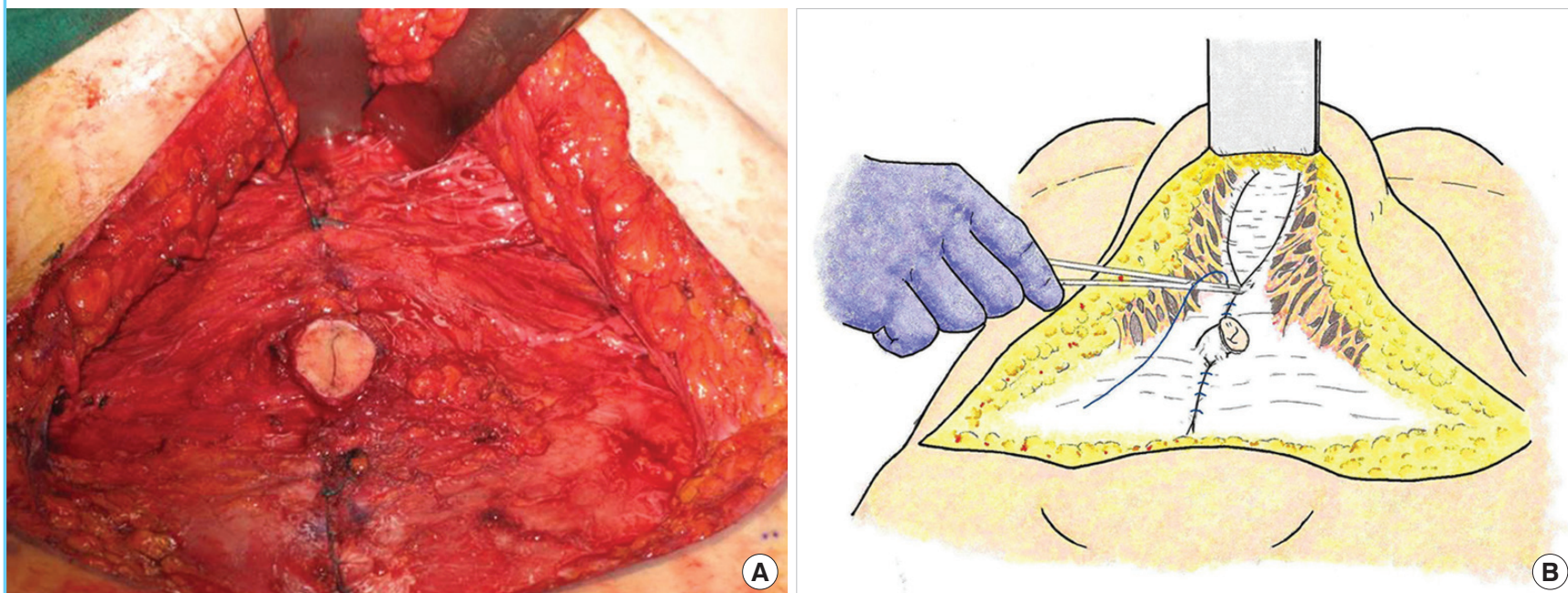
complications, and the patients had a flat abdomen and smooth waistline after surgery (Fig. 8).

The average follow-up period was 12.1 months (range, 2-40 months). Seventy-three patients had undergone natural childbirth, fifteen patients had undergone one Caesarian section, and nine patients had experienced two Caesarian sections. The average height of the patients was $161.12 \pm 4.65 \mathrm{~cm}$ and their average body weight was $55.59 \pm 7.56 \mathrm{~kg}$. On the basis of the 2000 World Health Organization Western Pacific Regional Office criteria [4], most patients had a body mass index within the normal range (mean, $21.50 \pm 2.95 \mathrm{~kg} / \mathrm{m}^{2}$ ). The average weight of the resected abdominal tissues was $194.88 \pm 139.99 \mathrm{~g}$, and the average dimensions of the excised skin were $21.78 \pm 6.77 \mathrm{~cm} \times$
$12.81 \pm 4.16 \mathrm{~cm}$, with an area of $279.00 \pm 136.93 \mathrm{~cm}^{2}$. The average infiltration volume of the tumescent solution was $1,646.91$ $\pm 699.41 \mathrm{~mL}$, and the volume of liposuction was $1,384.86 \pm$ $884.78 \mathrm{~mL}$.

Twenty-two patients experienced complications. One patient $(0.88 \%)$ suffered from a localized wound infection that was resolved by conservative treatment. Skin necrosis was observed in one patient $(0.88 \%)$, who required serial debridement and vacuum-assisted closure. Additional liposuction was performed in two undercorrected patients (1.77\%). Nine patients (7.96\%) with hypertrophic scars underwent scar revision. Seromas occurred in nine patients (7.96\%). Serial needle aspiration and compressive dressing were performed in two of the nine patients.

\section{Fig. 5. Schematic illustration of deep-plane lipoabdominoplasty}

(A) Preoperative status (blue line, Scarpa's fascia). (B) Liposuction was performed over the abdomen. (C) The lower abdominal tissue was removed.

(D) Abdominal flap sliding was performed without difficulty.
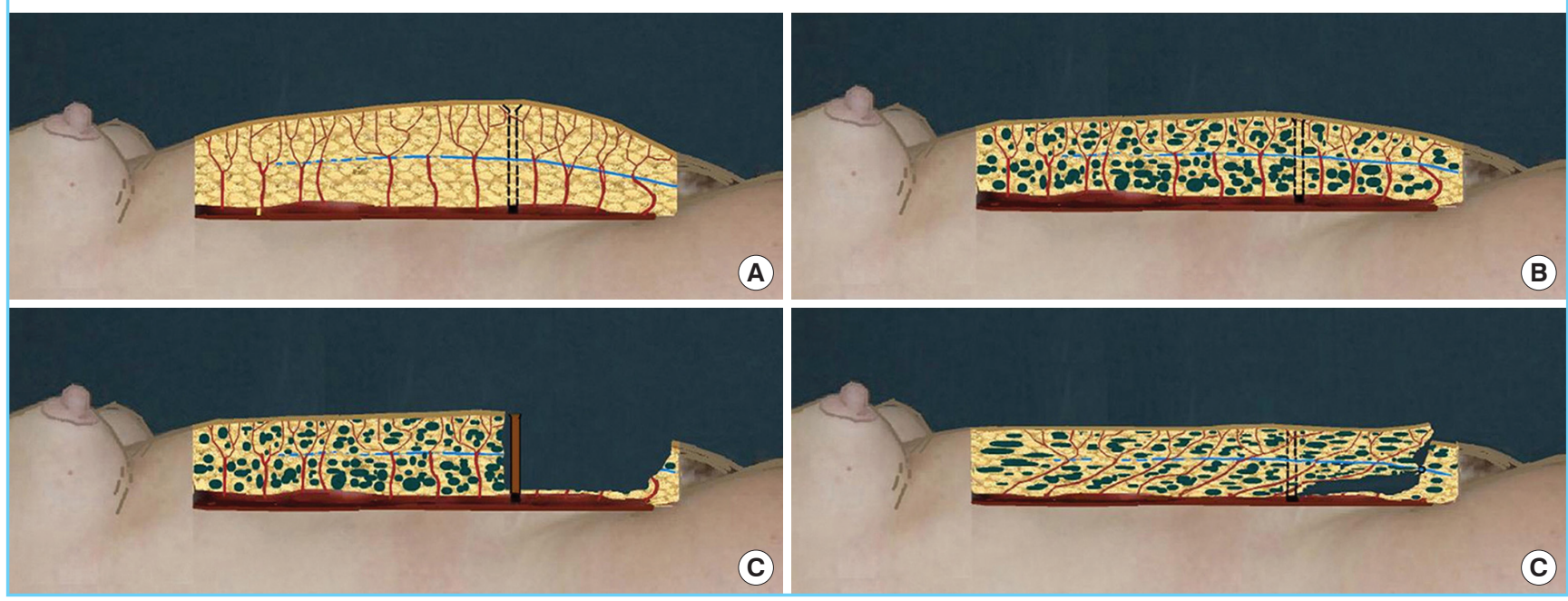

\section{Fig. 6. Scarpa's fascia repair}

The Scarpa's fascia was repaired first. It relieved skin tension and made dermal circulation intact.

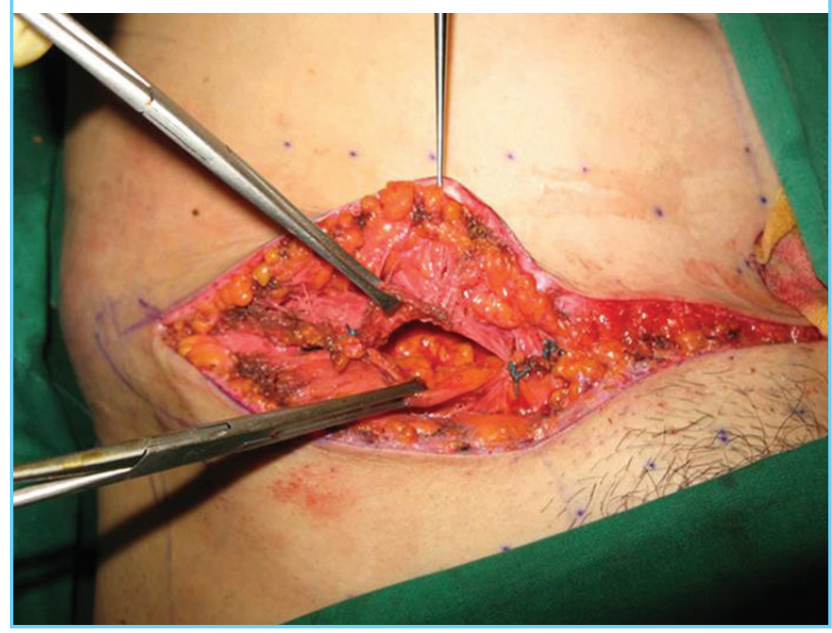

\section{Fig. 7. Skin closure using histoacryl}

After finishing dermal suture, skin bond (Histoacryl, B. Braun, Aesculap, Germany) was applied along the incisional wounds.

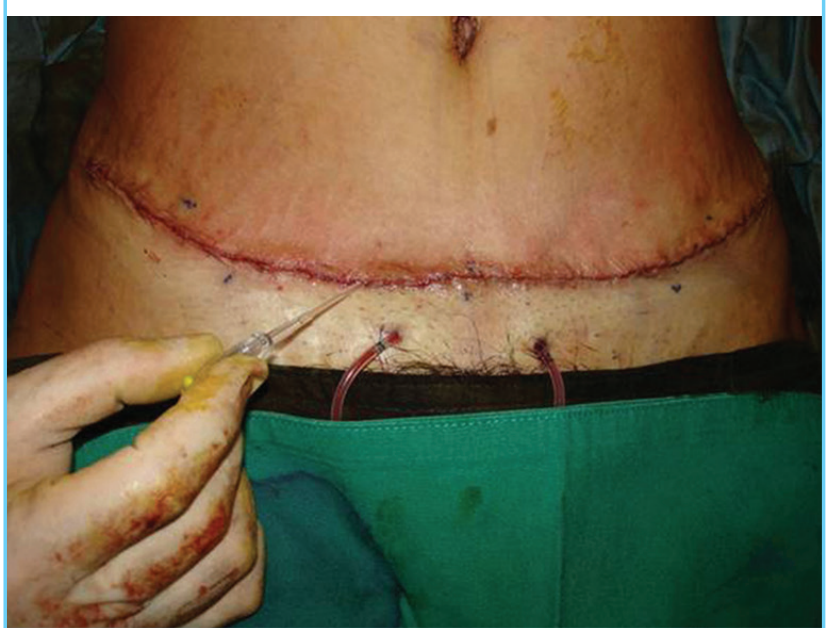




\section{Fig. 8. Case}

A 42-year-old woman with bulging and striae gravidarum underwent deep-plane lipoabdominoplasty. (A) Before surgery. (B) After surgery.
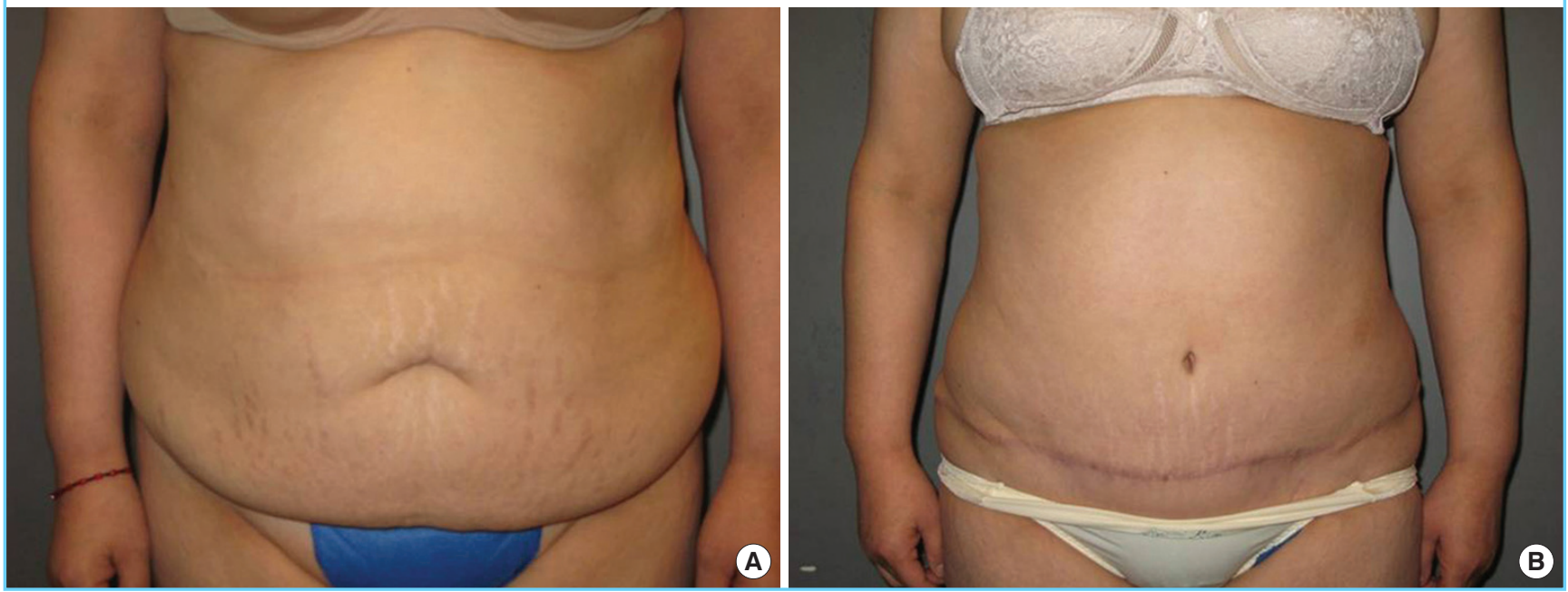

In the other seven patients, fibrin glue (Tisseel-Fibrin Sealant, Baxter AG, Vienna, Austria) was used for hemostasis, and a Jackson-Pratt drain was reapplied. The complications were successfully treated within seven days. No severe complications, such as deep-vein thrombosis or pulmonary embolism, occurred.

\section{DISCUSSION}

\section{History of the extent of undermining and the use of Scarpa's fascia}

Abdominoplasty consists of pannus excision, muscular tightening, and skin redraping for an hourglass waistline. The history of abdominoplasty began in the late 1960s.

Vernon successfully standardized the use of extensive undermining to allow easy flap mobilization and umbilical transposition [5]. After Illouz [6] developed the liposuction technique, the evolution of abdominoplasty accelerated. In 1985, Hakme [7] introduced the mini-abdominoplasty with liposuction, and beginning in 1991, Matarasso [8] recommended safe areas of abdominal circulation and dissection in his articles. In 1992, Illouz [6], Avelar [9], and Shestak [10] suggested advanced techniques of minimal to zero undermining accompanied by liposuction. In 1995, Lockwood [11] reported the high lateral tension abdominoplasty (HLTA) using Scarpa's fascial closure to decrease the tension of the abdominal flap. According to Rosenfield's [12] revision of the HLTA, Scarpa's fascial closure makes it possible to retract the skin more effectively and to remove the excess skin aggressively, especially in the central abdomen. Saldanha first reported the use of lipoabdominoplasty combined with aggressive liposuction and mobilization of the superficial abdominal tissue in 2002.

\section{Concept of the DPLA technique}

Extensive and aggressive liposuction without undermining Traditional abdominoplasty has an area of limited dissection or liposuction in order to avoid the deterioration of flap circulation, as Matarasso [8] argued. Abdominal undermining makes it difficult to reduce the thickness or excessive skin in the upper abdomen. Many surgeons currently perform a limited midline dissection during abdominoplasty. However, the area of limited dissection includes many central perforators from the deep epigastric vessels, especially in the periumbilical area (Fig. 9). It is critical to avoid dissecting the anterior surface of the rectus abdominis muscle as much as possible. Instead of undermining, we applied extensive and aggressive liposuction over the entire abdominal portion of the rectus muscle. Dissection was performed only over the area of diastasis recti. This made it possible to save the majority of the abdominal perforators and to mobilize the abdominal flap through the deep plane. Additionally, it led to an absence of dead space for seroma formation in the epigastrium.

Through the liposuction of superficial and deep fat, abdominal thickness can be managed while maintaining the abdominal perforators, especially from the deep epigastric vessels. This makes the abdomen a multi-pedicled neurovascular flap. After removal of superficial and deep fat, a sufficient sliding space is prepared, which is called 'mesh undermining.'

In fact, in the epigastric area, Scarpa's fascia is not definite. Monoplane premuscular liposuction is therefore likely to be performed in this area. In the umbilical area, however, it is possible to distinguish between the deep and superficial planes by the clear presence of Scarpa's fascia [13]. 


\section{Fig. 9. Preservation of the abdominal perforators}

(A) We were able to preserve many central perforators (the area marked in green) by dissecting the anterior surface of the rectus abdominis muscle to the minimal extent possible. (B) Intraoperative photographs of the limited dissection of the abdominal flap.

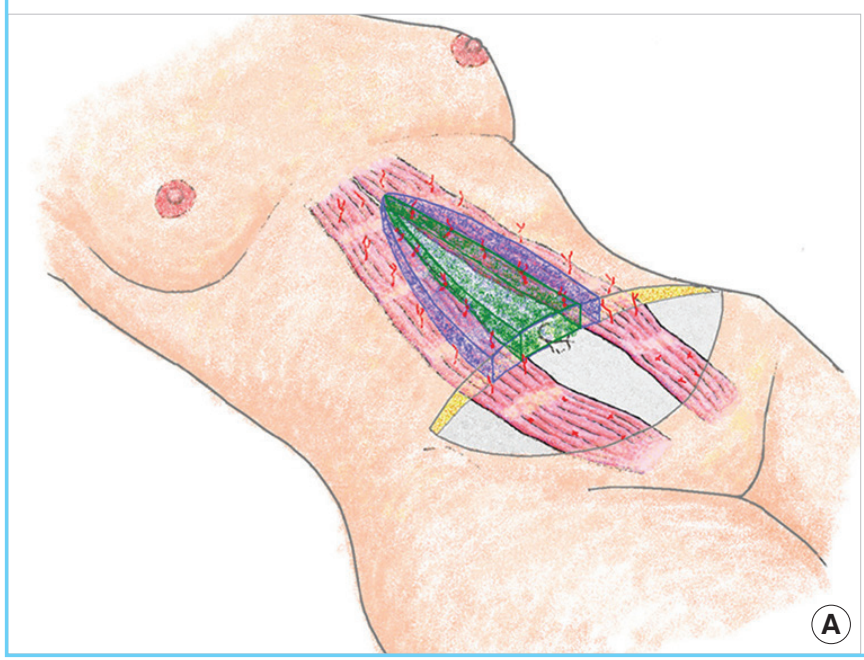

Plication of the rectus fascia

Rectus diastasis was repaired during surgery, and the medial borders of the rectus sheath were approximated without incising the sheath (Fig. 4). A continuous suture was placed midway between the xiphoid and pubis. It is important not to overlap to secure the plication, because the rate of recurrence of diastasis is higher in overcorrected cases [14].

Resection of the abdominal ellipse and sliding of the abdominal flap

Recently, Saldanha's lipoabdominoplasty has shown outstanding achievements in abdominal plastic surgery. On first glance, DPLA appears similar to Saldanha's technique, but significant differences are present between these two techniques with regard to tissue removal and mobilization of the abdominal flap.

Saldanha stated that the superficial liposuction technique introduced by De Souza Pinto is one of the fundamental pillars of lipoabdominoplasty, because it provides more mobility to the abdominal flap so that it can slide down easily and reach the suprapubic region. In his technique, the infraumbilical superficial tissue is removed, and the superficial tissue of the upper abdomen beyond Scarpa's fascia is lowered $[12,15]$.

In DPLA, the removal of the lower abdominal tissue from the skin to the deep fat layer, including Scarpa's fascia, takes place in an en bloc pattern, which makes it possible to mobilize the flap above the deep fascia and to manipulate Scarpa's fascia.

When the lower abdominal excess is removed, it is important for a certain amount of deep fat attached to the deep fascia to be preserved, especially in the bilateral femoral regions (* in Fig. 3). This preserves important lymphatic channels to help drain the

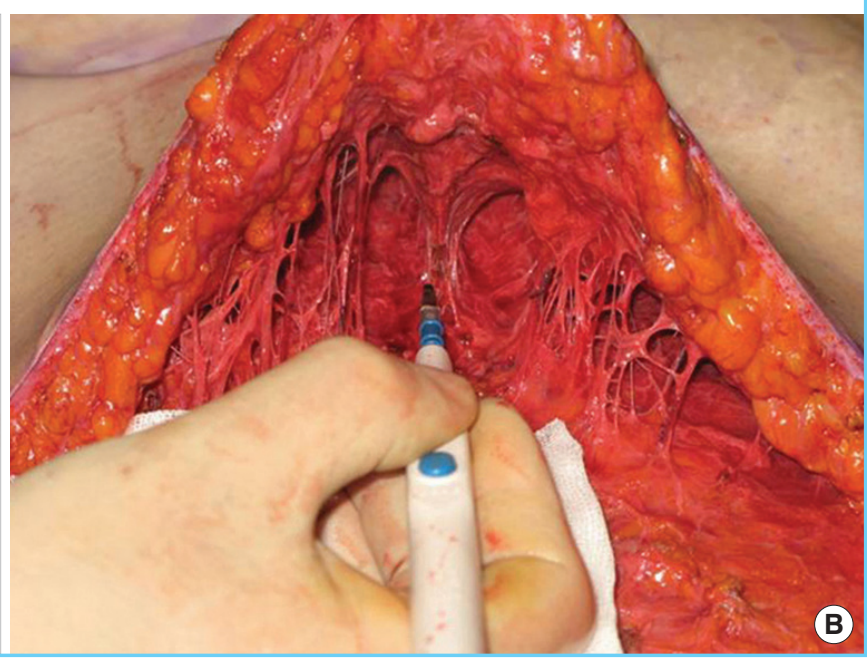

lower abdomen region and reduce seroma and edema formation $[16,17]$.

Moreover, it is easier to plicate diastasis recti through the defect after the excision of deep tissue than when Scarpa's fascia and deep fat remain present. The rectus plication procedure can be helpful in producing an hourglass-shaped waistline.

As a result of liposuction, the deep fat layer acts as the deep plane, between the rectus fascia and the Scarpa's fascia, and as the sliding surface (Fig. 5). The skin can be easily approximated so that it is not necessary to flex the hip. The repair of Scarpa's fascia plays an important role in this procedure for multiple reasons. Through fascial closure, the tension is relieved and the disturbance of dermal circulation is solved. Moreover, it allows high lateral tension abdominoplasty to be performed, since skin tension is created in the lateral region, rather than in the central region, as in traditional abdominoplasty. This aspect of the HLTA technique is helpful for securing the circulation of the low-central skin of the flap and for removing a sufficient amount of upper abdominal skin [12].

Repairing Scarpa's fascia can lead to the scar developing in a lower position [12]. Patients currently often show a preference for low-cut clothing, and the scar must therefore be hidden under the clothes, which requires a lower incision. During preoperative marking, it can be helpful for the patient to wear underwear or his or her preferred clothing. After wound closure, the lower abdominal skin is slightly pulled upward, which moves the scar to a higher location. This may be more severe when Scarpa's fascia is not repaired, because the skin is the only tension-bearing structure. Suspending Scarpa's fascia limits scar migration. Additionally, the resulting lower tension of the central region of 


\section{Table 1. DPLA compared with conventional abdominoplasty}

\begin{tabular}{|lll|}
\hline DPLA & Characteristic & $\begin{array}{c}\text { Conventional } \\
\text { abdominoplasty }\end{array}$ \\
\hline None/selective & Undermining & Wide \\
Low & Morbidity & High \\
Short & Operation time & Long \\
Fast & Recovery & Slow \\
Few & Complications & Many \\
Aggressive & Liposuction extent & Conservative \\
Minimal & Sensory change & Extensive \\
Low & Incision level & High \\
\hline
\end{tabular}

DPLA, deep-plane lipoabdominoplasty.

Table 2. DPLA compared with Saldanha's lipoabdominoplasty

\begin{tabular}{|lll|}
\hline DPLA & Characteristic & $\begin{array}{c}\text { Saldanha's } \\
\text { lipoabdominoplasty }\end{array}$ \\
\hline Remove & Scarpa fascia & Preserve \\
Low & Morbidity & Lower \\
Low & Incision level & High \\
Easy & Fascial management & Difficult \\
No & Fascial gathering & Yes \\
Maintain & Fascial tightening & Weak \\
\hline DPLA, deep-plane lipoabdominoplasty. & \\
\hline
\end{tabular}

the flap prevents superior displacement of the pubic area. The upward migration of the pubic hair and the mons pubis has an unfavorable aesthetic effect, especially in East Asians, who tend to have broader pubic regions than Caucasians.

In 2008, Rangaswamy [18] reported on a new lipoabdominoplasty technique that was similar to DPLA. However, DPLA is different from Mohan's technique in several ways. First, liposuction was performed on both the superficial and deep layers of the fat. Liposuction from the superficial to the deep plane further increases the mobility of the flap and reduces abdominal fat volume relative to liposuction only on the deep plane. Second, flap elevation was performed with the minimal width, which was limited to between the medial border of the rectus abdominis muscle in order to spare the abdominal perforator. Third, plication was carried out from the inferior to the superior side. This cleared the visual field for delicate manipulation. Finally, we resected the full layer of the lower abdominal tissue including Scarpa's fascia and the deep fat layer. This reduced the fullness around the closed abdominal wound more than Mohan's technique [13].

Compared with traditional abdominoplasty, DPLA has many advantages, such as a shorter surgical time and recovery time, less flap morbidity, and less sensory disturbance with fewer complications (Table 1). In comparison with Saldanha's lipoabdominoplasty, DPLA has several advantages, such as a lower incision level, easier musculofascial manipulation, the absence of fascial overlapping, and a long-term fascial tightening effect (Table 2). Using DPLA, we were able to correct abdominal deformities more effectively with fewer complications in comparison with traditional abdominoplasty.

\section{REFERENCES}

1. Stuerz K, Piza H, Kinzl JF. The impact of abdominoplasty after massive weight loss: a qualitative study. Ann Plast Surg 2013;71:547-9.

2. Stewart KJ, Stewart DA, Coghlan B, et al. Complications of 278 consecutive abdominoplasties. J Plast Reconstr Aesthet Surg 2006;59:1152-5.

3. Saldanha OR, De Souza Pinto EB, Mattos WN Jr, et al. Lipoabdominoplasty with selective and safe undermining. Aesthetic Plast Surg 2003;27:322-7.

4. Anuurad E, Shiwaku K, Nogi A, et al. The new BMI criteria for asians by the regional office for the western pacific region of WHO are suitable for screening of overweight to prevent metabolic syndrome in elder Japanese workers.J Occup Health 2003;45:335-43.

5. Vernon $S$. Umbilical transplantation upward and abdominal contouring in lipectomy. Am J Surg 1957;94:490-2.

6. Illouz YG. A new safe and aesthetic approach to suction abdominoplasty. Aesthetic Plast Surg 1992;16:237-45.

7. Hakme F. Technical details in the lipoaspiration associate with liposuction. Rev Bras Cir 1985;75:331-7.

8. Matarasso A. Abdominolipoplasty: a system of classification and treatment for combined abdominoplasty and suctionassisted lipectomy. Aesthetic Plast Surg 1991;15:111-21.

9. Avelar JM. Abdominoplasty without panniculus undermining and resection: analysis and 3-year follow-up of 97 consecutive cases. Aesthet Surg J 2002;22:16-25.

10. Shestak KC. Marriage abdominoplasty expands the miniabdominoplasty concept. Plast Reconstr Surg 1999;103: 1020-31.

11. Lockwood T. High-lateral-tension abdominoplasty with superficial fascial system suspension. Plast Reconstr Surg 1995; 96:603-15.

12. Rosenfield LK. High tension abdominoplasty 2.0. Clin Plast Surg 2010;37:441-65.

13. Kumar P, Pandey AK, Kumar B, et al. Anatomical study of superficial fascia and localized fat deposits of abdomen. Indian J Plast Surg 2011;44:478-83.

14. Nahas FX, Ferreira LM. Concepts on correction of the musculoaponeurotic layer in abdominoplasty. Clin Plast Surg 2010;37:527-38. 
15. Saldanha OR, Federico R, Daher PF, et al. Lipoabdominoplasty. Plast Reconstr Surg 2009;124:934-42.

16. Al A, Silvia CR, Albert C. Abdominoplasty. In: Guyuron B, Chung KC, editors. Plastic surgery: indications and practice. Philadelphia: Elsevier Saunders; 2009. p.1609-26.

17. Costa-Ferreira A, Rebelo M, Vasconez LO, et al. Scarpa fas- cia preservation during abdominoplasty: a prospective study. Plast Reconstr Surg 2010;125:1232-9.

18. Rangaswamy M. Lipoabdominoplasty: a versatile and safe technique for abdominal contouring. Indian J Plast Surg 2008;41(Suppl):S48-55. 